\title{
Degenerate Asymptotic Perturbation Theory
}

\author{
W. Hunziker and C. A. Pillet \\ Institut für Theoretische Physik, ETH-Hönggerberg, CH-8093 Zürich, Switzerland
}

\begin{abstract}
Asymptotic Rayleigh-Schrödinger perturbation theory for discrete eigenvalues is developed systematically in the general degenerate case. For this purpose we study the spectral properties of $m \times m$-matrix functions $A(\kappa)$ of a complex variable $\kappa$ which have an asymptotic expansion $\Sigma A_{k} \kappa^{k}$ as $\kappa \rightarrow 0$. We show that asymptotic expansions for groups of eigenvalues and for the corresponding spectral projections of $A(\kappa)$ can be obtained from the set $\left\{A_{k}\right\}$ by analytic perturbation theory. Special attention is given to the case where $A(\kappa)$ is Borel-summable in some sector originating from $\kappa=0$ with opening angle $>\pi$. Here we prove that the asymptotic series describe individual eigenvalues and eigenprojections of $A(\kappa)$ which are shown to be holomorphic in $S$ near $\kappa=0$ and Borel summable if $A_{k}^{*}=A_{k}$ for all $k$. We then fit these results into the scheme of Rayleigh-Schrödinger perturbation theory and we give some examples of asymptotic estimates for Schrödinger operators.
\end{abstract}

\section{Introduction}

Convergent (or analytic) perturbation theory has long been established in full generality by Kato [9] and Baumgärtel [3]. Yet, among the simplest problems of quantum mechanics, there are cases where the perturbation series diverge and have only an asymptotic validity: the anharmonic oscillator [10], the Stark-effect [7] and the Zeeman-effect [2]. In all these examples the perturbed eigenvalues can be constructed as the Borel-sum of their divergent asymptotic series.

These beautiful results are not quite satisfactory in one respect: they suffer from the unnatural assumption that the unperturbed eigenvalue is non-degenerate (or, in some cases, that its degeneracy is lifted in first order). In their accounts of asymptotic perturbation theory Reed and Simon [10] tacitly ignore the degenerate case while Kato ([9], Chap. 8) only discusses low order perturbations of semi-simple eigenvalues. Our aim is to remove restrictions of this kind and to develop asymptotic perturbation theory in the general degenerate case to any possible order.

Not surprisingly it turns out that the heart of the matter is the finite-dimensional case. Given an $m \times m$-matrix valued function $A(\kappa)$ of a complex variable $\kappa$ which 
has an asymptotic expansion

$$
A(k)=\sum_{k=0}^{N} A_{k} \kappa^{k}+o\left(\kappa^{N}\right) \equiv B(\kappa)+o\left(\kappa^{N}\right)
$$

as $\kappa \rightarrow 0$, what can we say about the asymptotic behaviour of its eigenvalues and eigenprojections? This problem is examined in Sects. 1 and 2. We show that the (convergent) perturbation series calculated from $B(\kappa)$ are asymptotic to groups of eigenvalues and to the corresponding spectral projections of $A(\kappa)$ up to a certain order which can be estimated in terms of $m$ and $N$. In Sect. 3 this is applied to functions $A(\kappa)$ which have an asymptotic expansion to all orders in $\kappa$. Then the eigenvalues of $A(\kappa)$ fall into groups which are asymptotically degenerate to all orders as $\kappa \rightarrow 0$. These groups and the corresponding spectral projections have asymptotic Puiseux-and Puiseux/Laurent-series which can be constructed from the set $\left\{A_{k}\right\}$ of the expansion coefficients by analytic perturbation theory. Of special importance to quantum mechanics is the case where $A_{k}^{*}=A_{k}$ for all $k$ (even when $A(\kappa)$ is not defined or not selfadjoint for real $\kappa)$; then the perturbation series reduce to asymptotic Taylor series.

In Sect. 4 we consider matrix-functions $A(\kappa)$ which are Borel-summable in some sector $S$ of opening angle $>\pi$ originating at $\kappa=0$. We show that the eigenvalues and eigenprojections of $A(\kappa)$ are holomorphic in $S$ near $\kappa=0$ and that different eigenvalues cannot be asymptotically degenerate to all orders as $\kappa \rightarrow 0$. The asymptotic series constructed in Sect. 3 then describe individual eigenvalues and eigenprojections of $A(\kappa)$. If $A_{k}^{*}=A_{k}$ for all $k$, we prove that the asymptotic Taylorseries are Borel-summable to the eigenvalues and eigenprojections and that the eigennilpotents vanish identically in $S$ near $\kappa=0$. For Borel-summable $A(\kappa)$ we thus obtain considerably more spectral information near $\kappa=0$ than in the general asymptotic case. This may be useful even if one is not actually interested in calculating and summing the perturbation series.

Section 5 contains an outline of asymptotic Rayleigh-Schrödinger perturbation theory in Hilbert space for degenerate discrete eigenvalues. The first requirement is that the eigenvalue under consideration is stable in the sense of Kato ([9], Chap. 8, Sect. 1). This is not discussed further, but we remark that non-perturbative methods are available to prove stability [11]. The standard reduction then leads to an operator $A(\kappa)$ acting in the finite-dimensional unperturbed spectral subspace. Rayleigh-Schrödinger perturbation theory gives an expansion of $A(\kappa)$ in powers of $\kappa$, and the second problem is to estimate its $N^{\text {th }}$ order remainder. We give some illustrations of asymptotic estimates for Schrödinger operators using the method of exponential bounds. The third step is then to construct asymptotic expansions for the eigenvalues and spectral projections of $A(\kappa)$ : this is where our results for the finite-dimensional case apply.

\section{Asymptotics of Eigenvalues}

With $C^{m}$ we denote the $m$-dimensional complex Hilbert space. Then $L\left(C^{m}\right)$ is the algebra of linear operators on $C^{m}$ with the corresponding operator norm. 
Theorem 1.1 Let $S$ be a complex set with $0 \in \bar{S} \backslash S$ and $A(\kappa)$ a function from $S$ to $L\left(C^{m}\right)$, which has an asymptotic expansion in powers of $\kappa$ to some order $N \geqq 0$ :

$$
\begin{aligned}
& A(\kappa)=\sum_{k=0}^{N} A_{k} \kappa^{k}+C(\kappa) \equiv B(\kappa)+C(\kappa), \\
& \lim _{\kappa \rightarrow 0}\left\|\kappa^{N} C(\kappa)\right\|=0 .
\end{aligned}
$$

Then

(i) the Puiseux-series for the eigenvalues of $B(\kappa)$ are asymptotic to the eigenvalues of $A(\kappa)$ to all orders $\leqq N / m$;

(ii) if $A_{k}^{*}=A_{k}$ for $k=0 \ldots N$, the Taylor-series for the eigenvalues of $B(\kappa)$ are asymptotic to the eigenvalues of $A(\kappa)$ to all orders $\leqq N$.

Remarks. According to analytic perturbation theory ([3], [9]) the eigenvalues of $B(\kappa)$ form a set of cycles $\{b(\kappa)\}$ : each cycle is a different multivalued analytic function

$$
\begin{aligned}
b(\kappa) & =\sum_{k=0}^{\infty} b_{k} \kappa^{k}, \\
k & =r / p ; \quad r=0,1,2 \ldots
\end{aligned}
$$

in a punctured disc $0<|\kappa|<\delta$, where $p$ is the order of the branch point $\kappa=0$ of $b(\kappa)$. Each cycle represents a certain number $g$ of eigenvalues which are equally distributed among the $p$ branches: $g$ is a multiple of $p$ and each branch of $b(\kappa)$ is an eigenvalue of $B(\kappa)$ with algebraic multiplicity $g / p$. We call $g$ the "weight" and $p$ the "length" of the cycle $b(\kappa)$.

For any $n \geqq 0$ the " $n^{\text {th }}$ order eigenvalues" of $B(\kappa)$ are given by the cycles

$$
b^{(n)}(\kappa)=\sum_{k \leq n} b_{k} \kappa^{k}
$$

obtained from (1.2) by dropping all terms of order $>n$. In this process several "parent" cycles $b(\kappa)$ may reduce to the same cycle $b^{(n)}(\kappa)$. The length $p^{(n)}$ of $b^{(n)}(\kappa)$ is then a common divisor of the lengths of the parents and the weight $g^{(n)}$ the total weight of the parents. Each branch of $b^{(n)}(\kappa)$ represents $g^{(n)} / p^{(n)}$ eigenvalues of $B(\kappa)$ up to errors of order $o\left(|\kappa|^{n}\right)$. For $r<n$ the same reduction process leads from $\left\{b^{(n)}(\kappa)\right\}$ to $\left\{b^{(r)}(\kappa)\right\}$ : we indicate this relation by writing

$$
\left\{b^{(r)}(\kappa)\right\}<\left\{b^{(n)}(\kappa)\right\} .
$$

The precise statement of Theorem 1.1 (i) is :if $n \leqq N / m$, then each branch of $b^{(n)}(\kappa)$ also describes $g^{(n)} / p^{(n)}$ eigenvalues of $A(\kappa)$ up to errors of order $o\left(|\kappa|^{n}\right)$ as $\kappa \rightarrow 0$ in $S$.

If $B^{*}(\kappa)=B(\kappa)$ for real $\kappa$ (where $A(\kappa)$ need not be defined) the eigenvalues of $B(\kappa)$ are holomorphic in a disc $|\kappa|<\delta([9]$, Chap. II, Theorem 1.10). Then (1.2) reduces to the Taylor-series $(p=1)$ for an eigenvalue $b(\kappa)$ of algebraic multiplicity $g$. Part (ii) of Theorem 1.1 says that for $n \leqq N, b^{(n)}(\kappa)$ also represents a group of $g^{(n)}$ eigenvalues of $A(\kappa)$ up to errors of order $o\left(|\kappa|^{n}\right)$ as $\kappa \rightarrow 0$ in $S$.

The proof of Theorem 1.1 will be based on the following estimates: 
Lemma 1.2. Let $A=B+C$ be a sum in $L\left(C^{m}\right)$ and $\Gamma$ a circle in the complex plane. If $\left\|(z-B)^{-1} C\right\|<1$ for all $z \in \Gamma$, then $A$ and $B$ have the same number of eigenvalues (including algebraic multiplicities) inside $\Gamma$.

Proof. Let $A(\lambda)=B+\lambda C,|\lambda| \leqq 1$. Then

$$
(z-A(\lambda))^{-1}=\left(1+\lambda(z-B)^{-1} C\right)^{-1}(z-B)^{-1}
$$

exists for all $z \in \Gamma$ and the spectral projection

$$
P(\lambda)=(2 \pi i)^{-1} \oint_{\Gamma} d z(z-A(\lambda))^{-1}
$$

is continous in $\lambda$ for $|\lambda| \leqq 1$. Therefore $\operatorname{dim} P(0)=\operatorname{dim} P(1)$

\section{Lemma 1.3.}

$$
\left\|A^{-1}\right\| \leqq|\operatorname{det} A|^{-1}\|A\|^{m-1}
$$

for all $A \in L\left(C^{m}\right)$ with $\operatorname{det} A \neq 0$.

Proof. A has a polar decomposition $A=R U$ with $U$ unitary and $R=R^{*}>0$. Let $r_{1} \leqq r_{2} \ldots \leqq r_{m}$ be the eigenvalues of $R$. Then $0<r_{1}$ and (1.5) follows since $\left\|A^{-1}\right\|$ $=r_{1}^{-1},|\operatorname{det} A|=r_{1} r_{2} \ldots r_{m}$ and $\|A\|=r_{m}$

Using (1.5) to estimate $\left\|(z-B)^{-1}\right\|$ in Lemma 1.2 we obtain:

Lemma 1.4. Let $A=B+C$ be a sum in $L\left(C^{m}\right), b_{1} \ldots b_{m}$ the eigenvalues of $B$ and $\Gamma$ a circle in the complex plane. If

$$
\prod_{h=1}^{m}\left|z-b_{h}\right|>\|z-B\|^{m-1}\|C\|
$$

for all $z \in \Gamma$, then $A$ and $B$ have the same number of eigenvalues (including algebraic multiplicities) inside $\Gamma$.

Proof of Theorem 1.1. For convenience we describe the eigenvalues of $B(\kappa)$ by $m$ single-valued functions $b_{1}(\kappa) \ldots b_{m}(\kappa)$ over some simply connected region (e.g. $0<|\kappa|$ $<\delta, 0<\arg \kappa \leqq \pi$ ). We fix $n$ in $0 \leqq n \leqq N / m$. Then

$$
b_{h}(\kappa)=b_{h}^{(n)}(\kappa)+o\left(|\kappa|^{n}\right),
$$

where $b_{h}^{(n)}(\kappa)$ are the $n^{\text {th }}$ order eigenvalues. If $b_{h}^{(n)}(\kappa) \equiv b_{k}^{(n)}(\kappa)$, we say that $h \sim k$. If $h \neq k$ it follows from (1.3) that

$$
\left|b_{h}^{(n)}(\kappa)-b_{k}^{(n)}(\kappa)\right| \geqq \beta|\kappa|^{n}
$$

for some $\beta>0$ and small $\kappa$. Let $\Gamma_{\kappa}$ be the circle with center $b_{1}^{(n)}(\kappa)$ and radius $2 R|\kappa|^{n}$, where $R$ is chosen in $0<R<\beta / 4$. For $|\kappa|$ sufficiently small we see from (1.6) and (1.7) that $b_{h}(\kappa)$ is inside or outside $\Gamma_{\kappa}$ for $h \sim 1$ or $h \nsim 1$ and has distance $>R|\kappa|^{n}$ from $\Gamma_{\kappa}$ in both cases. Since $n m \leqq N$, it follows from (1.1) that

$$
\prod_{h=1}^{m}\left|z-b_{h}(\kappa)\right| \geqq R^{m}|\kappa|^{n m}>\|z-B(\kappa)\|^{m-1}\|C(\kappa)\|
$$


for small $\kappa \in S$ and all $z \in \Gamma_{\kappa}$. By Lemma $1.4 A(\kappa)$ and $B(\kappa)$ then have the same number of eigenvalues inside $\Gamma_{\kappa}$. Repeating this argument we locate all eigenvalues of $A(\kappa)$ within circles of radius $2 R|\kappa|^{n}$ around the $n^{\text {th }}$ order eigenvalues $b_{h}^{(n)}(\kappa)$, provided that $|\kappa|$ is sufficiently small (depending on $R$ ). Part (i) of the theorem now follows since $R$ may be chosen arbitrarily small.

To prove part (ii) we recall that in this case $B(\kappa)$ has a spectral representation

$$
B(\kappa)=\sum_{h} b_{h}(\kappa) P_{h}(\kappa)
$$

with holomorphic eigenvalues $b_{h}(\kappa)$ and eigenprojections $P_{h}(\kappa)$ in a disc $|\kappa|<\delta([9]$, Chap. II, Theorem 1.10). Hence there is a constant $\gamma$ such that

$$
\left\|(z-B(\kappa))^{-1}\right\| \leqq \gamma\left(\min _{h}\left|z-b_{h}(\kappa)\right|\right)^{-1} .
$$

Using this bound instead of (1.5) we obtain the following variant of Lemma 1.4: if

$$
\min _{h}\left|z-b_{h}\right|>\gamma\|C\|
$$

for all $z \in \Gamma$, then $A$ and $B$ have the same number of eigenvalues inside $\Gamma$. The proof of part (i) is modified accordingly: we fix $n$ in $0 \leqq n \leqq N$, and find

$$
\min _{h}\left|z-b_{h}(\kappa)\right| \geqq R|\kappa|^{n}>\gamma\|C(\kappa)\|
$$

for small $\kappa \in S$ and all $z \in \Gamma_{\kappa}$. By (1.8) $A(\kappa)$ and $B(\kappa)$ then have the same number of eigenvalues inside $\Gamma_{\kappa}$

\section{Asymptotics of Spectral Projections}

We assume that $A(\kappa)$ satisfies the hypothesis of Theorem 1.1. For $n \leqq N / m$ and a given cycle $b^{(n)}(\kappa)$ we call

$$
\begin{gathered}
P_{B}(\kappa)=(2 \pi i)^{-1} \oint_{\Gamma_{\kappa}} d z(z-B(\kappa))^{-1} \quad \text { and } \\
P_{A}(\kappa)=(2 \pi i)^{-1} \oint_{\Gamma_{\kappa}} d z(z-A(\kappa))^{-1}
\end{gathered}
$$

the spectral projections of $B(\kappa)$ and $A(\kappa)$ corresponding to the common $n^{\text {th }}$ order eigenvalues $b^{(n)}(\kappa)$. Here $\Gamma_{\kappa}$ is any one of the circles of radius $2 R|\kappa|^{n}$ constructed in the proof of Theorem 1.1 around the $p^{(n)}$ values of $b^{(n)}(\kappa)$. Therefore $P_{B}(\kappa)$ and $P_{A}(\kappa)$ are $p^{(n)}$-valued functions, defined for small $\kappa \in C(\kappa \neq 0)$ and small $\kappa \in S$, respectively.

As a result of analytic perturbation theory ([9], Chap. II, Theorem 1.8) $P_{B}(\kappa)$ is analytic in a punctured disc $0<|\kappa|<\delta$ with a branch point of order $p^{(n)}$ at $\kappa=0$. This branch point may be an algebraic pole of order $\leqq n(m-1)$ so that $P_{B}(\kappa)$ has a convergent Puiseux/Laurent-series

$$
\begin{aligned}
P_{B}(\kappa) & =\sum_{k} P_{k} \kappa^{k}, \\
k & =r / p^{(n)}, \quad r=s, s+1, \ldots,
\end{aligned}
$$


where $s$ is a possibly negative integer $\geqq-n(m-1) p^{(n)}$. In fact $s<0$ always occurs when $p^{(n)}>1$ ([9], Chap. II, Theorem 1.9).

In the special case of Theorem 1.1 (ii) it suffices to choose $n \leqq N$. Then $\left\|P_{B}(\kappa)\right\|=1$ for real $\kappa$ which implies $s \geqq 0$ and therefore $p^{(n)}=1$. In this case (2.1) reduces to a Taylor-series.

Theorem 2.1. Suppose that $A(\kappa)$ satisfies the hypothesis of Theorem 1.1. Let $P_{B}(\kappa)$ and $P_{A}(\kappa)$ be the spectral projections of $B(\kappa)$ and $A(\kappa)$ corresponding to a common $n^{\text {th }}$ order eigenvalue, where $n \leqq N / m$ in general and $n \leqq N$ in the special case $A_{k}^{*}=A_{k}$ $(k=0 \ldots N)$. Then

(i) the Puiseux Laurent-series of $P_{B}(\kappa)$ is asymptotic to $P_{A}(\kappa)$ to all orders $\leqq N-n(2 m-1)$;

(ii) if $A_{k}^{*}=A_{k}$ for $k=0 \ldots N$, the Taylor-series of $P_{B}(\kappa)$ is asymptotic to $P_{A}(\kappa)$ to all orders $\leqq N-n$.

Proof. Choosing $\kappa \in S$ sufficiently small, we have $\left\|(z-B(\kappa))^{-1} C(\kappa)\right\|<1 / 2$ for all $z \in \Gamma_{\kappa}$, and therefore

$$
\left\|(z-A(\kappa))^{-1}-(z-B(\kappa))^{-1}\right\|<2\left\|(z-B(\kappa))^{-1}\right\|^{2}\|C(\kappa)\| .
$$

As in the proof of Theorem 1.1 we use the estimates

$$
\left\|(z-B(\kappa))^{-1}\right\|<\mathrm{const}|\kappa|^{-q},
$$

with $q=m n$ in case (i) and $q=n$ in case (ii). Since $\Gamma_{\kappa}$ has length $\sim|\kappa|^{n}$, we obtain from (1.1), (2.2), (2.3),

$$
\left\|P_{A}(\kappa)-P_{B}(\kappa)\right\|=o\left(|\kappa|^{N}\right)|\kappa|^{n-2 q}
$$

Remark. The estimates given in Theorems 1.1 and 2.1 can easily be improved if more than (1.7) is known about the splitting of $n^{\text {th }}$ order eigenvalues.

\section{Asymptotic Series}

Theorem 3.1. Let $S$ be a complex set with $0 \in \bar{S} \backslash S$, and $A(\kappa)$ a function from $S$ to $L\left(C^{m}\right)$ which has an asymptotic power-series

$$
A(\kappa) \sim \sum_{k=0}^{\infty} A_{k} \kappa^{k} \quad(k \text { integer })
$$

for $\kappa \rightarrow 0$. Then $A(\kappa)$ has the following spectral properties for small $\kappa:$

(i) the eigenvalues form a set of cycles $\{a(\kappa)\}$ : each cycle is given by a different formal Puiseus-series

$$
a(\kappa): \sum_{k=0}^{\infty} a_{k} \kappa^{k}, \quad k=r / p, r=0,1,2 \ldots, p=\lim _{n \rightarrow \infty} p^{(n)},
$$

where $p^{(n)}$ is the number of branches of the finite part

$$
a^{(n)}(\kappa)=\sum_{k \leqq n} a_{k} \kappa^{k}
$$

Each cycle has a weight $g=$ integer multiple of $p$, and each branch of $a(\kappa)$ is an asymptotic series for a group of $g / p$ eigenvalues of $A(\kappa)$ which are degenerate to all orders in $\kappa$; 
(ii) the spectral projections of $A(\kappa)$ corresponding to these $p$ groups form a p-valued function $P(\kappa)$ which has an asymptotic Puiseux/Laurent-series

$$
P(\kappa) \sim \sum_{k=s / p}^{\infty} P_{k} \kappa^{k}, \quad k=r / p, \quad r=s, s+1, s+2 \ldots,
$$

where $s$ is a possibly negative integer ( $s<0$ always occurs when $p>1)$;

(iii) if $A_{k}^{*}=A_{k}$ for all $k$, then the series (3.2) and (3.4) reduce to Taylor-series $(p=1, s=0)$;

(iv) the finite parts of (3.2) and (3.4) are obtained from the finite parts

$$
A^{(N)}(\kappa)=\sum_{k=0}^{N} A_{k} \kappa^{k}
$$

by analytic perturbation theory as described in the proof.

Proof. Let $\left\{a^{(n)}(\kappa)\right\}$ be the $n^{\text {th }}$ order eigenvalues of $A^{(N)}(\kappa)$ for $N \geqq n m$. By Theorem 1.1 they are independent of $N$ and therefore satisfy $\left\{a^{(r)}(\kappa)\right\}<\left\{a^{(n)}(\kappa)\right\}$ if $r<n$, in the sense of (1.4). Hence there exists $v<\infty$ such that for $n \geqq v$ the number of cycles $a^{(n)}(\kappa)$ as well as the length $p^{(n)}=p$ and the weight $g^{(n)}=g$ of each cycle are independent of $n$. Then each $a^{(n)}(\kappa)$ is the finite part of a different cycle (3.2) with length $p$ and weight $g$. For $n \geqq v$ the $n^{\text {th }}$ order eigenvalues $a^{(n)}(\kappa)$ are separated by distances $>\beta|\kappa|^{v}$ for some $\beta>0$, and by Theorem 1.1 each branch of $a^{(n)}(\kappa)$ describes a group of $g / p$ eigenvalues of $A(\kappa)$ up to errors of order $o\left(|\kappa|^{n}\right)$. Since $n$ is arbitrarily large it follows that two eigenvalues in the same group have a distance vanishing faster than any power of $\kappa$ as $\kappa \rightarrow 0$. This proves (i).

To prove (ii) we note that $P(\kappa)$ are the spectral projections of $A(\kappa)$ corresponding to the $v^{\text {th }}$ order eigenvalues $a^{(v)}(\kappa)$ which $A(\kappa)$ has in common with $A^{(N)}(\kappa)$ for $N \geqq v m$. Let $P^{(N)}(\kappa)$ be the corresponding spectral projections of $A^{N}(\kappa)$. By Theorem 2.1 we obtain the expansion (3.4) to any desired order $n$ by choosing $N \geqq n+v(2 m-1)$ and expanding $P^{(N)}(\kappa)$ to all orders $\leqq n$. This proves (ii) and (iv). Part (iii) is then evident from the second parts of Theorems 1.1 and 2.1

Remarks. The essence of Theorem 3.1 is that the eigenvalues of $A(\kappa)$ fall into groups whose diameter in the complex plane vanishes faster than any power of $\kappa$ and which are separated by distances $>\beta|\kappa|^{v}$ for some $\beta>0$ and $v<\infty$. These groups and the corresponding spectral projections have asymptotic expansions to all orders in $\kappa$ which are obtained from the expansion of $A(\kappa)$ by analytic perturbation theory.

In the following section we introduce a class of families $A(\kappa)$ for which different eigenvalues cannot be degenerate to all orders in $\kappa$. Then the series (3.2) and (3.4) are asymptotic to the individual eigenvalues and eigenprojections of $A(\kappa)$.

\section{Borel-summability}

\section{Definitions}

(i) Throughout this section $S$ is a fixed sector $S=\{\kappa \in C|0<| \kappa \mid, \alpha<\arg \kappa<$ $\alpha+\beta\}$ with opening angle $\beta$ in $\pi<\beta<2 \pi$. For any $\delta>0$, we define $S_{\delta}=\{\kappa \in S \mid$ $|\kappa|<\delta\}$.

(ii) $f \in B^{m}$ means: there exist $\delta>0, C, \sigma$ such that $f$ is a holomorphic function 
from $S_{\delta}$ to $L\left(C^{m}\right)$, which has an asymptotic power-series

$$
f(\kappa) \sim \sum_{k=0}^{\infty} f_{k} \kappa^{k} \quad(k \text { integer })
$$

satisfying

$$
\left\|f_{N}(\kappa)\right\| \leqq C \sigma^{N} N \text { ! }
$$

for all $\kappa \in S_{\delta}$ and all $N \in Z^{+}$, where $f_{N}(\kappa)$ is given by

$$
f(\kappa)=\sum_{k=0}^{N-1} f_{k} \kappa^{k}+f_{N}(\kappa) \kappa^{N} .
$$

(iii) Let $f \in B^{m}$. Then $f^{*} \sim f$ means that $f_{k}^{*}=f_{k}$ for all $k$.

Remarks. The functions $f \in B^{m}$ are called "Borel-summable" since they can be reconstructed uniquely from the expansion coefficients $\left\{f_{k}\right\}$ (see e.g. [10], Theorem XII. 21). In particular $f(\kappa) \equiv 0$ if $f \in B^{m}$ and $f_{k}=0$ for all $k$. We also note that $f+g \in B^{m}$ and $f g \in B^{m}$ if $f, g \in B^{m}$.

Theorem 4.1. If $A \in B^{m}$, then there exist constants $\varepsilon>0, \beta>0,0 \leqq n<\infty$ such that

(i) on $S_{\varepsilon}$, the eigenvalues of $A(\kappa)$ and the corresponding eigenprojections are given by holomorphic functions $a_{1}(\kappa) \ldots a_{s}(\kappa)$ and $P_{1}(\kappa) \ldots P_{s}(\kappa)$, respectively;

(ii) $\left|a_{h}(\kappa)-a_{k}(\kappa)\right|>\beta|\kappa|^{n}$

for all pairs $h \neq k$ and all $\kappa \in S_{\varepsilon}$.

Remark. It follows from (ii) that for $A \in B^{m}$ the asymptotic series given by Theorem 3.1 refer to individual eigenvalues and eigenprojections.

Proof of Theorem 4.1. Since $A(\kappa)$ is holomorphic in $S_{\delta}$ the number $s$ of different eigenvalues of $A(\kappa)$ is constant in $S_{\delta}$ unless $\kappa$ is an exceptional point where this number is $<s$. These points cannot accumulate in $S_{\delta}$. In any simply connected subdomain of $S_{\delta}$ containing no exceptional point, the eigenvalues and eigenprojections of $A(\kappa)$ are given by holomorphic functions $a_{1}(\kappa) \ldots a_{s}(\kappa)$ and $P_{1}(\kappa) \ldots P_{s}(\kappa)$, respectively. In particular each eigenvalue $a_{h}(\kappa)$ has constant multiplicity $g_{h}=\operatorname{dim} P_{h}(\kappa)$. For a proof of these statements we refer to [9], Chap. II, Sect. 1.

To prove (i) it therefore suffices to show that exceptional points cannot accumulate at $\kappa=0$. On $C^{m} \otimes C^{m}$ we define

$$
F(\kappa)=A(\kappa) \otimes 1-1 \otimes A(\kappa) .
$$

The eigenvalues of $F(\kappa)$ are the differences of the eigenvalues of $A(\kappa)$. In particular, zero is an eigenvalues of $F(\kappa)$ with algebraic multiplicity

$$
q=g_{1}^{2}+g_{2}^{2}+\cdots+g_{s}^{2}
$$

for nonexceptional $\kappa$. Thus $F(\kappa)$ has a characteristic polynomial

$$
\begin{aligned}
P(z, \kappa) & =z^{q} \sum_{k=0}^{m^{2}-q} f_{k}(\kappa) z^{k} \quad \text { with } \\
f_{0}(\kappa) & \neq 0 \quad \text { for nonexceptional } \kappa .
\end{aligned}
$$


If $\kappa$ is exceptional, then zero is an eigenvalue of $F(\kappa)$ with algebraic multiplicity $>q$, hence

$$
f_{0}(\kappa)=0 \quad \text { for exceptional } \kappa
$$

Next we note that $f_{0} \in B^{1}$ since $f_{0}$ is a polynomial of matrix-elements of $A(\kappa)$. Now suppose that $\kappa_{n} \rightarrow 0$ is a sequence of exceptional points. Then $f_{0}\left(\kappa_{n}\right)=0$ by (4.4), which implies that $f_{0}$ has vanishing expansion coefficients. Since $f \in B^{1}$, it follows that $f_{0}(\kappa)=0$ for all $\kappa \in S_{\delta}$, in contradiction to (4.3). This proves (i).

To prove (ii) suppose that for some pair $h \neq k, d(\kappa)=a_{h}(\kappa)-a_{k}(\kappa)$ vanishes faster than any power of $\kappa$ as $\kappa \rightarrow 0$. By part (i) of the proof we know that $d(\kappa) \neq 0$ for small $\kappa$, so that $d(\kappa)$ is a zero of $z^{-q} P(z, \kappa)$ :

$$
f_{0}(\kappa)=-\sum_{k=1}^{m^{2}-q} f_{k}(\kappa) d(\kappa)^{k}
$$

This shows that $f_{0}(\kappa)$ vanishes faster than any power of $\kappa$ as $\kappa \rightarrow 0$. Since $f_{0} \in B^{1}$, it follows again that $f_{0}(\kappa) \equiv 0$ in $S_{\delta}$, in contradiction to (4.3)

Theorem 4.2. Suppose that $A \in B^{m}$ and $A^{*} \sim A$. Then the eigenvalues and eigenprojections of $A(\kappa)$ are Borel-summable and the eigennilpotents vanish identically for small $\kappa$.

Remark. Since the sector $S_{\delta}$ has opening angle $>\pi$, it always contains a piece of the real axis. We emphasize two points: $S_{\delta}$ need not be symmetric with respect to the real axis, and $A^{*} \sim A$ does not mean that $A(\kappa)$ is necessarily selfadjoint for real $\kappa$.

The proof of Theorem 4.2 is prepared by the following two lemmas:

Lemma 4.3. Suppose that $f \in B^{m}$ and that $f_{0}^{-1}$ exists. Let $g(\kappa)=f(\kappa)^{-1}$. Then $g \in B^{m}$. In particular $g$ satisfies the asymptotic estimate

$$
\left\|g_{N}(\kappa)\right\| \leqq C\left(1+C^{2}\right)^{N} \sigma^{N} N !
$$

in any sector $S_{\delta}$ where

$$
\begin{gathered}
\|g(\kappa)\| \leqq C \quad \text { and } \\
\left\|f_{N}(\kappa)\right\| \leqq C \sigma^{N} N !
\end{gathered}
$$

Proof. Inequalities (4.6) and (4.7) hold in some $S_{\delta}$ since $f(\kappa)^{-1}$ exists if $\left\|f(\kappa)-f_{0}\right\|<$ $\left\|f_{0}^{-1}\right\|^{-1}$. Then $g(\kappa)=f(\kappa)^{-1}$ is holomorphic in $S_{\delta}$ so that it remains to derive (4.5) from (4.6), (4.7). For $N \geqq 1$ all terms of order $\geqq N$ in the identity

must cancel:

$$
1=\sum_{k=0}^{N-1} f(\kappa) g_{k} \kappa^{k}+f(\kappa) g_{N}(\kappa) \kappa^{N}
$$

$$
0=\sum_{k=0}^{N-1} f_{N-k}(\kappa) g_{k}+f(\kappa) g_{N}(\kappa), \text { i.e. } \quad g_{N}(\kappa)=-g(\kappa) \sum_{k=0}^{N-1} f_{N-k}(\kappa) g_{k},
$$

which for $\kappa \rightarrow 0$ gives the recursion for $g_{N}$. By (4.6), (4.7),

$$
\left\|g_{N}(\kappa)\right\| \leqq C^{2} \sum_{k=0}^{N-1}\left\|g_{k}\right\| \sigma^{N-k}(N-k) !
$$


for $N \geqq 1$, while for $N=0$

$$
\left\|g_{0}(\kappa)\right\|=\|g(\kappa)\| \leqq C .
$$

From (4.8)-(4.10) and using $(N-k) ! k ! \leqq(N-1) !(1 \leqq k \leqq N-1)$, one confirms by induction that for $N \geqq 1$

$$
\left\|g_{N}(\kappa)\right\| \leqq C\left(C^{2}+C^{4}+\cdots+C^{2 N}\right) \sigma^{N} N !
$$

Lemma 4.4. Suppose that $A \in B^{m}$. Let a be an eigenvalue of $A_{0}$ and $\Pi(\kappa)$ the spectral projection of $A(\kappa)$ for the a-group (i.e. the group of eigenvalues $a_{h}(\kappa) \rightarrow$ a as $\kappa \rightarrow 0$ ). Then $\Pi \in B^{m}$.

Proof. Analyticity of $\Pi(\kappa)$ in $S_{\varepsilon}$ follows from Theorem 4.1. For a fixed circle $\Gamma$ around a we expand

$$
\Pi(\kappa)=(2 \pi i)^{-1} \oint_{\Gamma} d z R(z, \kappa)
$$

by expanding $R(z, \kappa)=(z-A(\kappa))^{-1}$ in powers of $\kappa$. Since $R(z, \kappa)$ is uniformly bounded for all $z \in \Gamma$ and small $\kappa$, it follows from Lemma 4.3 that

$$
\left\|R_{N}(z, \kappa)\right\| \leqq \text { const } \sigma^{N} N \text { ! }
$$

for some $\delta(\Gamma)>0$ and all $(z, \kappa) \in \Gamma \times S_{\delta}$. Hence $\left\|\Pi_{N}(\kappa)\right\| \leqq$ const $\sigma^{N} N$ !

Proof of Theorem 4.2. We consider a single eigenvalue

$$
a(\kappa)=\sum_{k=0}^{N-1} a_{k} \kappa^{k}+a_{N}(\kappa) \kappa^{N}
$$

of $A(\kappa)$ and the corresponding eigenprojection $P(\kappa)$ which are both holomorphic in $S_{\varepsilon}$ (Theorem 4.1) and have asymptotic Taylor-series with real and symmetric coefficients, respectively (Theorem 3.1). By (4.2) the other eigenvalues of $A(\kappa)$ have distances $>\beta|\kappa|^{n}$ from $a(\kappa)$ with $\beta>0$ and integer $n \geqq 0$. We may assume that

$$
a_{0}=a_{1}=\cdots=a_{n+1}=1
$$

by adding to $A(\kappa)$ a suitable polynomial multiple of the identity. Let $\Pi_{1}(\kappa)$ be the spectral projection for thel-group of $A(\kappa)$ and

$$
A_{1}(\kappa)=\kappa^{-1}(A(\kappa)-1) \Pi_{1}(\kappa) .
$$

Then $\kappa A_{1}(\kappa) \in B^{m}$ by Lemma 4.4 and

$$
\lim _{\kappa \rightarrow 0} \kappa A_{1}(\kappa)=\left(A_{0}-1\right) \Pi_{1}(0)=0,
$$

since $A_{0}^{*}=A_{0}$. It follows that $A_{1} \in B^{m}$ and that $A_{1}^{*} \sim A_{1}$, since $\Pi_{1}^{*} \sim \Pi_{1}$ by Theorem 2.1 .

Any eigenvalue $b_{1}(\kappa) \neq 0$ of $A_{1}(\kappa)$ defines an eigenvalue $b(\kappa)=1+\kappa b_{1}(\kappa)$ of $A(\kappa)$ with the same eigen projection. Conversely, $a_{1}(\kappa)$ as defined by (4.11) is an eigenvalue of the 1-group of $A_{1}(\kappa)$ since $a_{1}=1$. Repeating this argument we arrive at

$$
a(\kappa)=1+\kappa+\kappa^{2}+\cdots+\kappa^{n}+\kappa^{n+1} a_{n+1}(\kappa),
$$


where $a_{n+1}(\kappa)$ is an eigenvalue of the 1-group of

$$
\begin{gathered}
A_{n+1}(\kappa)=\kappa^{-1}\left(A_{n}(\kappa)-1\right) \Pi_{n+1}(\kappa), \\
\Pi_{n+1}(\kappa)=\text { spectral projection for the } \\
1 \text {-group of } A_{n}(\kappa),
\end{gathered}
$$

which satisfies $A_{n+1} \in B^{m}$ and $A_{n+1}^{*} \sim A_{n+1}$. Any eigenvalue $b_{n+1}(\kappa) \neq 0$ of $A_{n+1}(\kappa)$ defines an eigenvalue

$$
b(\kappa)=1+\kappa+\kappa^{2}+\cdots+\kappa^{n}+\kappa^{n+1} b_{n+1}(\kappa)
$$

of $A(\kappa)$ with the same eigenprojection. From (4.2), (4.12), (4.13), it follows that $a(\kappa)=b(\kappa)$ for all $\kappa \in S_{\varepsilon}$. Therefore $a_{n+1}(\kappa)$ is the only eigenvalue $\neq 0$ of $A_{n+1}(\kappa)$ and $P(\kappa)$ is the spectral projection for the 1-group of $A_{n+1}(\kappa)$. This proves $P \in B^{m}$ (Lemma 4.4). Also,

$$
a_{n+1}(\kappa)=(\operatorname{dim} P(\kappa))^{-1} \operatorname{trace}\left(A_{n+1}(\kappa)\right),
$$

which shows that $a_{n+1} \in B^{1}$ and therefore $a \in B^{1}$.

The eigennilpotent $D(\kappa)=(A(\kappa)-a(\kappa)) P(\kappa)$ satisfies

$$
D(\kappa)^{m}=0, \quad D \in B^{m}, \quad D^{*} \sim D .
$$

From $D(\kappa)^{m}=D_{0}^{m}+o(\kappa)$ we see that $D_{0}^{m}=0$, hence $D_{0}=0$ since $D_{0}^{*}=D_{0}$. Therefore $D_{1}(\kappa)=\kappa^{-1} D(\kappa)$ also satisfies (4.14). Proceeding in this way we conclude that $D(\kappa)$ has vanishing expansion coefficients. Since $D \in B^{m}$, it follows that $D(\kappa)=0$ for all $\kappa \in S_{\varepsilon}$

\section{Rayleigh-Schrödinger Perturbation Theory}

Here we describe briefly how our results for the finite-dimensional case fit into the scheme of RS-perturbation theory in Hilbert space. Essentially we follow [10], but we also include some ideas which have since been developed in connection with the Zeeman- and Stark-effect ([2], [7]). No formal proofs will be given.

Let $A(\kappa)$ be a family of closed operators on a Hilbert space $\mathscr{H}$, defined for $\kappa$ in some complex set $S \ni 0$, and $R(z, \kappa)=(z-A(\kappa))^{-1}$ its resolvent. We define

$$
\begin{gathered}
\Delta=\{z \in C \mid \text { for small } \kappa \in S, R(z, \kappa) \text { exists and is } \\
\text { bounded uniformly in } \kappa\} .
\end{gathered}
$$

A discrete eigenvalue $\lambda$ of $A_{0}=A(0)$ with eigen projection $P_{0}$ is said to be stable with respect to the family $A(\kappa)$ if

(i) $\{z|0<| z-\lambda \mid<\varepsilon\} \subset \Delta$

for some $\varepsilon>0$, and

(ii) $\lim _{\kappa \rightarrow 0}\left\|P(\kappa)-P_{0}\right\|=0$ for

$$
P(\kappa)=(2 \pi i)^{-1} \oint_{\Gamma} d z R(z, \kappa),
$$

where $\Gamma$ is a circle of radius $<\varepsilon$ around $\lambda$. 
Generally speaking, $\lambda$ will be stable under mild continuity conditions for $A(\kappa)$ as $\kappa \rightarrow 0$, provided that $\lambda$ is separated from the essential spectrum of $A(\kappa)$ for small $\kappa[11]$. Here $P(\kappa)$ is the spectral projection for the $\lambda$-group $\lambda_{h}(\kappa)=\lambda+\Delta \lambda_{h}(\kappa)$, where $\Delta \lambda_{h}(\kappa)$ are the eigenvalues of $\Delta A(\kappa)=P(\kappa)(A(\kappa)-\lambda) P(\kappa)$ considered as an operator on $M(\kappa)=\operatorname{Ran} P(\kappa)$. For small $\kappa$ this can be transformed into an equivalent eigenvalue problem in the space $M_{0}=\operatorname{Ran} P_{0}$ by any linear map $S(\kappa)$ from $M(\kappa)$ onto $M_{0}$. A possible choice is shown in Fig. 1:

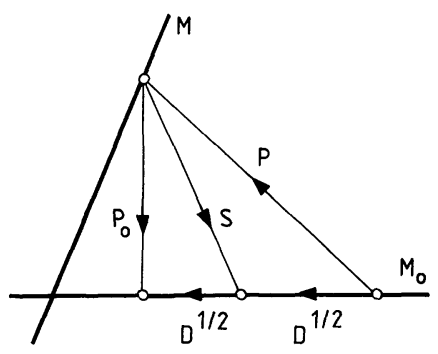

Fig. 1 .

Here we consider

$$
D(\kappa)=P_{0} P(\kappa) P_{0}
$$

as an operator on $M_{0}$. By (5.2) we have $\|D(\kappa)-1\|<1$ for small $\kappa$ so that $D^{-1}$ and $D^{-1 / 2}$ are well defined. Then the operator

$$
S(\kappa)=D(\kappa)^{-1 / 2} P_{0} P(\kappa)
$$

from $M(\kappa)$ to $M_{0}$ has the inverse

$$
S(\kappa)^{-1}=P(\kappa) P_{0} D(\kappa)^{-1 / 2},
$$

and the eigenvalue problem for $\Delta A(\kappa)$ is equivalent to the eigenvalue problem for

$$
\begin{aligned}
E(\kappa) & =S(\kappa) \Delta A(\kappa) S(\kappa)^{-1} \\
& =D(\kappa)^{-1 / 2} N(\kappa) D(\kappa)^{-1 / 2}, \quad \text { where } \\
N(\kappa) & =P_{0} P(\kappa)(A(\kappa)-\lambda) P(\kappa) P_{0}
\end{aligned}
$$

is again operating on $M_{0}$. The eigenprojections and eigennilpotents of $E(\kappa)$ and $A(\kappa)$ are also related by the similarity transformation (5.5). A more judicious but less explicit choice of $S(\kappa)$ is given by Kato ([9], Chap. II, Sect. 4). RS-perturbation theory provides expansions of $E(\kappa)$ and $S(\kappa)$ for the case $A(\kappa)=A_{0}+\kappa V$. From the iterated resolvent equation

$$
R=\sum_{k=0}^{N-1} \kappa^{k} R_{0}\left(V R_{0}\right)^{k}+\kappa^{N} R\left(V R_{0}\right)^{N}
$$

and (5.3), (5.4), we find, for example,

$$
\begin{aligned}
D(\kappa) & =\sum_{k=0}^{N-1} D_{k} \kappa^{k}+D_{N}(\kappa) \kappa^{N}, \quad \text { with } \\
D_{N}(\kappa) & =(2 \pi i)^{-1} \oint_{\Gamma} d z P_{0} R\left(V R_{0}\right)^{N} P_{0},
\end{aligned}
$$


and $D_{k}=D_{k}(0)$. A similar expansion holds for $N(\kappa)$, with an extra factor $(z-\lambda)$ in the integral (5.7). Computing the expansion of $E(\kappa)$ from (5.6), we are then confronted with a problem of finite-dimensional asymptotic perturbation theory. In quantum mechanics we are mainly (but not exclusively) concerned with selfadjoint problems: $A_{0}^{*}=A_{0}$ and $V^{*}=V$, formally. Due to our choice of $S(\kappa)$ the ex pansion coefficients of $D(\kappa), N(\kappa)$ and $E(\kappa)$ will then be symmetric. This explains the particular attention given to this case in Sects. 1-4.

We now turn to asymptotic estimates. For a stable eigenvalue $R(z, \kappa)$ is uniformly bounded for $z \in \Gamma$ and small $\kappa$ by (5.1). From (5.7) we then see that $\left\|D_{N}(\kappa)\right\|$ is bounded by a constant times

$$
C_{N}=\sup _{z \in \Gamma}\left\|\left(V R_{0}(z)\right)^{N} P_{0}\right\|,
$$

in which $\kappa$ and the full resolvent $R(z, \kappa)$ no longer appear. The same bound occurs in the expansion of $N(\kappa)$. If $V R_{0}(z)$ is bounded for some $z$ we are back to analytic perturbation theory: stability is then automatic and the RS-series have a strictly positive radius of convergence.

Asymptotic perturbation theory deals with cases where $V R_{0}$ is unbounded: then $C_{N}<\infty$ can only hold due to special properties of $\operatorname{Ran} P_{0}$. We illustrate some of the possibilities with the example of the anharmonic oscillator

$$
A(\kappa)=p^{2}+x^{2}+\kappa V(x)
$$

on $\mathscr{H}=L^{2}\left(R^{v}\right)$, where $V(x)$ is a real and locally square-integrable function on $R^{v}$. If $V(x)$ is bounded from below, $A(\kappa)$ has a purely discrete spectrum for $\kappa$ in the sector

$$
S=\{\kappa \mid-\pi+\varepsilon<\arg \kappa<\pi-\varepsilon\} \quad(\varepsilon>0),
$$

and any eigenvalue $\lambda$ of $A_{0}=p^{2}+x^{2}$ is stable with respect to the family $A(\kappa), \kappa \in S$ [11]. Moreover, $A(\kappa)$ is an analytic family in $S \backslash\{0\}$. The eigenfunctions of $A_{0}$ are of the form $H(x) \exp \left(-x^{2} / 2\right)$, with $H(x)$ a polynomial, so that

$$
\left\|e^{\alpha x^{2}} P_{0}\right\|<\infty \text { for } \alpha<1 / 2 \text {. }
$$

A simple technique introduced by Combes and Thomas [4] allows us to derive such exponential bounds without explicit knowledge of the eigenfunctions:

Let $\lambda$ be a discrete eigenvalue of $A_{0}=p^{2}+W(x)$ with eigenprojection $p_{0}, f(x)$ a smooth real function on $R^{v}$ which is bounded from below, and

$$
A_{f}=e^{f} A_{0} e^{-f}=(p+i \nabla f)^{2}+W(x) \text {. }
$$

Then the resolvents of $A_{f}$ and $A_{0}$ are related by

$$
e^{-f} R_{f}(z)=R_{0}(z) e^{-f}
$$

for all $z$ in the intersection of the two resolvent sets. Therefore $A_{f}$ and $A_{0}$ have the same (discrete) spectrum in the complement of $\sigma_{\text {ess }}\left(A_{f}\right) \cup \sigma_{\text {ess }}\left(A_{0}\right)$ with corresponding eigenprojections related by

$$
e^{-f} P_{f}=P_{0} e^{-f} \text {. }
$$

Since $\operatorname{Ran} e^{-f}$ is dense and $\operatorname{dim} P_{0}<\infty$, it follows that $\operatorname{Ran} P_{0}$ is in the domain of $e^{f}$, 
i.e.

$$
\left\|e^{f} P_{0}\right\|<\infty \text {. }
$$

There exists a variety of methods to control $\sigma_{\text {ess. }}\left(A_{f}\right)([10]$, Sects. XIII. $4-5 ;[6]$; [11]) and thus to derive exponential bounds ([10], Sect. XIII. 11; [15]). Suppose now that $\lambda \notin \sigma_{e s s}\left(A_{s f}\right)$ for all $s$ in $0 \leqq \mathrm{~s} \leqq 1$. From (5.8), (5.12) and (5.13), we then obtain

$$
C_{N}=\sup _{z \in \Gamma}\left\|V e^{-f / N} R_{f / N} V e^{-f / N} R_{2 f / N} \ldots V e^{-f / N} R_{f} e^{f} P_{0}\right\|<\infty,
$$

if the function $V(x)^{N} e^{-f(x)}$ is bounded. Choosing $f(x)=\alpha x^{2}$ in the example $A_{0}=$ $p^{2}+x^{2}$, we find

$$
A_{f}=p^{2}+x^{2}\left(1-4 \alpha^{2}\right)+2 i \alpha(p x+x p)
$$

which has purely discrete spectrum for $0 \leqq \alpha<1 / 2$. Therefore, if $\lambda$ is any eigenvalue of $A_{0}$, the RS-series for the family (5.9) are asymptotic in the sector (5.10) to order $N$ as long as $V(x)^{N} \exp \left(-\alpha x^{2}\right)$ is bounded for some $\alpha<1 / 2$.

The model example for Borel-summability is (5.9) with $V(x)=x^{4}([10]$, Sect. XII. 4). With the choice of $f(x)$ given above $x^{2} R_{s f}$ is bounded uniformly for $z \in \Gamma$ and $0 \leqq s \leqq 1$ so that

$$
C_{N}<\text { const }\left(\sup _{x} x^{2} e^{-\alpha x^{2}}\right)^{N} N^{N}<C \sigma^{N} N !
$$

for suitable $C$ and $\sigma$. Since $A(\kappa)$ is an analytic family in $S \backslash\{0\}, P(\kappa)$ and $E(\kappa)$ are holomorphic in some $S_{\delta}=\{\kappa \in S|0<| \kappa \mid<\delta\}$. Therefore $E(\kappa)$ is Borel-summable and also satisfies $E^{*}(\kappa)=E(\kappa)$ for small real $\kappa>0$. Theorems 4.1 and 4.2 then describe the spectral properties of $E(\kappa)$ and of $\Delta A(\kappa)=S(\kappa)^{-1} E(\kappa) S(\kappa)$.

A fundamentally different situation arises when $V(x)$ in (5.9) is not bounded below, as in the example

$$
V(x)=x^{2} x_{1} x_{2}
$$

for $v \geqq 2$. Since the expansion coefficients of $E(\kappa)$ still exist and are symmetric, and since the estimate (5.15) remains unchanged, we might at first take Borelsummability for granted. In fact there is already a problem with the definition of $A(\kappa)$ for real $\kappa \neq 0$ as a selfadjoint operator. This is avoided by restricting $\kappa$ to a smaller sector

$$
S=\{\kappa \mid 0<\varepsilon<\arg \kappa<\pi-\varepsilon\} .
$$

Then $A(\kappa)$ is again an analytic family in $S \backslash\{0\}$ with purely discrete spectrum and all eigenvalues of $A_{0}$ are stable with respect to $A(\kappa)$ [11]. However, Borel-summability does not follow since $S$ has now opening angle $<\pi$. What is needed first is an analytic extension of $E(\kappa)$ to a larger sector with opening angle $>\pi$ where the RS-series is still asymptotic with an estimate of type (5.15). For special perturbations like (5.16) this can be achieved using the scaling properties of $A(\kappa)$ under complex dilations $x \rightarrow \mu x$, $\mu \in C$ ([1]; [10], Sect. XIII. 10). The extended function $E(\kappa)$ is then defined but no longer symmetric for small real $\kappa$. However, since the expansion coefficients of $E(\kappa)$ are symmetric, Theorems 4.1 and 4.2 still apply. For real $\kappa$ the eigenvalues of $E(\kappa)$ have imaginary parts vanishing faster than any power of $\kappa$ as $\kappa \rightarrow 0$. Presumably this 
phenomenon has an interpretation in terms of unstable states associated with the illdefined Hamiltonian $A(\kappa)$ for real $\kappa \neq 0$. Whatever this picture may be, the mathematical objects which we calculate by summing the RS-series (in a sector symmetric with respect to the imaginary axis) are clearly defined. A better understood example of this kind is the Stark-effect ([7], [8]).

Due to our results for the finite-dimensional case, restrictions on the degeneracy of the unperturbed problem are no longer necessary. In this respect asymptotic perturbation theory is now developed to the same level as analytic perturbation theory.

Acknowledgement. It is a pleasure to thank B. Simon for his comments on the first draft of this paper.

\section{References}

1. Aguilar, J., Combes, J. M.: A class of analytic perturbations for one-body Schrödinger Hamiltonians. Commun. Math. Phys. 22, 269-279 (1971)

2. Avron, J. E., Herbst, I. W., Simon, B.: Schrödinger operators with magnetic fields III. Atoms in homogeneous magnetic field. Commun. Math. Phys. 79, 529-572 (1981)

3. Baumgärtel, H.: Endlich-dimensionale analytische Störungs-theorie. Berlin: Akademie-Verlag 1972

4. Combes, J. M., Thomas, L.: Asymptotic behaviour of eigenfunctions for multiparticle Schrödinger operators. Commun. Math. Phys. 34, 251-270 (1973)

5. Deift, P., Hunziker, W., Simon, B., Vock, E.: Pointwise bounds of eigenfunctions and wave packets in $N$-Body quantum systems. Commun. Math. Phys. 64, 1-34 (1978)

6. Enss, V.: A note on Hunziker's theorem Commun. Math. Phys. 52, 233-238 (1977)

7. Herbst, I. W., Simon, B.: Dilation analyticity in constant electric field II. $N$-Body problem, Borelsummability. Commun. Math. Phys. 80, 181-216 (1981)

8. Herbst, I. W.: Temporal exponential decay for the Stark-effect in atoms. Stockholm: Mittag-Leffler Institute, Report No. 16 (1981)

9. Kato, T.: Perturbation theory for linear operators. Berlin, Heidelberg, New York: Springer 1966

10. Reed, M., Simon, B.: Methods of modern mathematical physics. IV. Analysis of operators. New York: Academic Press 1978

11. Vock, E., Hunziker, W.: Stability of Schrödinger eigenvalue problems. Commun. Math. Phys. 83, 281-302 (1982)

Communicated by B. Simon

Received January 20, 1983; in revised form March 7, 1983 
